\title{
Evaluating creativity in parametric design environments and Geometric modelling environments
}

\author{
Rongrong $\mathrm{Yu}^{\mathrm{a}}$, Ning $\mathrm{Gu}^{\mathrm{b}}$ and Michael Ostwald ${ }^{\mathrm{c}}$ \\ a School of Engineering and Built Environment, Griffith University, \\ Southport, Australia; ${ }^{b}$ School of Art, Architecture and Design, Uni- \\ versity of South Australia, Adelaide, Australia; ' School of Architec- \\ ture and Built Environment, The University of Newcastle, Callaghan, \\ Australia.
}

Correspondence details: Rongrong Yu, Address: G39 Room 3.30, Gold Coast Campus, Griffith University, Queensland 4222, Australia. Email: r.yu@griffith.edu.au

\begin{abstract}
This paper presents a cognitive study that evaluates design creativity in parametric design environments, in relation to more conventional geometric modelling environments. The study correlates the results of design outcome evaluations and design process analysis. To achieve this comparison, a combined method of jury evaluation and protocol analysis is adopted. Firstly, eight professional architects were asked to complete two architectural design tasks with similar complexity, respectively in a parametric design environment and a geometric modelling environment. Following the design experiment, 19 experts were then invited to evaluate the design
\end{abstract}


outcomes produced by designers using pre-defined criteria focusing on design creativity. The creativity evaluation criteria address concerns related to innovation, usefulness, and unexpectedness. Outcomes of the evaluation and comparison are correlated to findings of a series of protocol studies on related parametric design processes. Results of this study suggest that parametric design potentially enhances overall design creativity from various perspectives.

Keywords. Design creativity; Parametric design; Protocol study; Jury evaluation

\section{Introduction}

Creativity is a critical requirement in the design domain. The abundance of research on creativity indicates that it is a common concern in the field of design studies. In architecture, it is one of the key criteria used to judge the impression that a design makes. It triggers several critical determinants such as social influence and individual behaviours related to design processes (Csikszentmihalyi 1988). Recent evidence suggests that different design media can also play a part in supporting design creativity. For example, design creativity has been evaluated and compared in freehand and digital sketching environments (Tang, Lee, and Gero 2011), in blindfolded and freehand 
sketching environments (Bilda, Gero, and Purcell 2006; Kan and Gero 2007) and in freehand sketching and CAD environments (Bilda and Demirkan 2003). Kim and Maher (2005) have also compared design creativity associated with a Graphical User Interface and a Tangible User Interface. From such studies it is clear that the design environment potentially has an impact on the production of creative works. In recent years, parametric design approaches have increasingly been applied in architectural practice. Parametric design defines a language of design based on vocabularies such as geometries and grammars in the forms of rules and relationships (Jabi et al. 2017). According to Woodbury (2010), it supports the creation, management and organisation of complex digital design models. By changing the parameters to control variations, particular design instances can be created or altered from a potentially infinite range of solutions (Kolarevic 2003; Lawson 2002). Parametric design thinking reflects on an "exploration and re-editing process of associative relationships in a geometrical solution space” (Oxman 2017). Such research suggests that parametric design differs from conventional digital design methods (i.e. those supported in geometric modelling environments) both in terms of design processes and outcomes. Furthermore, one of 
the overarching interests in parametric design research is to determine whether or not parametric concepts, methods and environments may enhance or hinder designer’s creativity. A popular opinion voiced in the literature is that parametric methods and environments do help to promote creativity (Qian, Chen, and Woodbury 2007; Schnabel 2007; Iordanova et al. 2009; Lee et al. 2012). However, there is a lack of empirical evidence connecting parametric design processes and tools to the production of creative design outcomes. The fundamental question, which has thus far remained unanswered is, do certain patterns of design thinking in parametric environments promote or hinder creative outcomes? The present paper takes a preliminary step towards answering this question.

This paper is an extension of previously published research by the Authors comparing design cognition in parametric and geometric design environments, which focused on designers' cognitive behaviour (Yu et al. 2015; Yu, Ostwald, and Gu 2015). Shifting the focus away from design processes and behaviours in isolation, this paper focuses on and evaluates the outcomes produced in design experiments, to explore the correlation between design process and levels of 
creativity exhibited in a design outcome. That is, we evaluate the creativity evident in design outcomes produced during an early concept development stage, in both a Parametric Design Environment (PDE) and a Geometric Modelling Environment (GME). In our study, eight professional architects were asked to complete two architectural design tasks with similar complexity, respectively in a PDE and a GME. Following the design experiment, 19 experts evaluated the design outcomes produced in the experiments based on a set of pre-defined criteria associated with design creativity. Creativity in design can be characterised in terms of aesthetic appeal, novelty, quality, unexpectedness, and uncommonness (Runco and Pritzker 1999). Many researchers have proposed criteria of novelty, value and unexpectedness for evaluating design creativity (Rosenman and Gero 1993; Runco and Pritzker 1999). In this study we assess creativity in terms of "Overall creativity, Innovation, Usefulness, and Surprisingness.” The outcomes of the expert evaluation of the experiments are finally correlated to the protocol analysis results of the design processes used, in order to explore the relationship between parametric design processes and design creativity. 
The results of this research seek to enhance our understanding of the benefits and limits of parametric design and provide insights into triggers and activities during parametric design processes, that can support creative outcomes.

\section{Background}

\subsection{Digital design environments - PDE and GME}

It has been argued that design media affects designers' design processes (Chien and Yeh 2012; Mitchell 2003). Past research suggests that sketching can assist design thinking as an effective design medium (Black 1990; Schön 1983). In a similar way, with the increasing application of digital design tools, researchers have started to study the influence of digital design tools on design processes (Bilda and Demirkan 2003; Fallman 2003; Kim and Maher 2008).

In the late 1990s, with the growing adoption of CAD 3D modelling tools in the design industry, architects began to identify ways in which these tools were superior to earlier CAD 2D drafting systems. Typical 3D geometric modelling tools used in architecture today include ArchiCAD from Graphisoft, Revit from Autodesk, 
Rhino from McNeel and Maya and Sketchup. In the last decade a similar paradigm shift has begun to occur, with BIM and parametric design software beginning to challenge the role played by traditional 3D geometric modelling software in the architectural industry.

Oxman (2000) argues that the different media of design are knowledge-intensive computational environments. Designers share design knowledge that can be represented and employed in computational environments. Parametric design, as a computational design environment, supports a new way of thinking in architectural design, although its actual impact on designers and design processes have yet to be thoroughly explored.

In the present study, for the purposes of comparison, Rhino was chosen as the traditional Geometric Modelling Environment (GME). Grasshopper, a widely-used parametric plugin to Rhino, was provided to participants as the Parametric Design Environment (PDE). Grasshopper has a graphic interface, which makes the rule-setting process more friendly and intuitive for designers. Because of this advantage as well as its relative popularity amongst the parametric designers, Grasshopper was selected as a PDE for our experiments. It is 
a typical parametric design tool sharing the same principles of rule-algorithmic design with other mainstream parametric tools.

The combination of Rhino (GME) and Grasshopper (PDE) is also ideal for the present study as the former offers advanced freeform 3D modelling features that will not lead to significant differences between the outcomes of the two environments. In addition, Grasshopper is an add-on in Rhino, which means that the two design environments can be hosted on the same platform during experiments. This combination ensures that the comparative goals of the experiment are both more reasonable and achievable.

\subsection{Evaluating design creativity}

Creativity often involves generating or producing something that did not exist or was not known previously. However, different disciplines (such as psychology and the social sciences) and creative domains (such as architecture and industrial design) use different definitions of creativity. According to psychologists Runco and Prizker (1999), creativity is characterised as aesthetic appeal, novelty, quality, unexpectedness, uncommonness, peer-recognition, influence, intelligence, learning and popularity. To evaluate whether an artefact is creative or 
not is an important issue for scholars, designers and educators.

Kaufman and Sternberg (2006) claim that "creativity can be measured, at least in some degree” (2). However, the evaluation of creativity can be quite subjective, and therefore evaluation standards are not easily defined (Jordanous 2011).

Creativity in design shares some common characteristics with creativity in psychology, social psychology and cognition. To critically measure creativity, a key step is to establish a set of objective and comprehensive evaluation criteria. For instance, standardised methods such as Torrance Tests of Creative Thinking (TTCT) (Torrance 1966) have been used for evaluating students’ design ideas produced in PDEs (Iordanova et al. 2009). In architectural design, creativity can also be expressed through a purposeful problem solving process, rather than a random one (Lawson 1997). Therefore, design creativity is not just about the production of something new, but also something useful. Roseman and Gero define a creative product as one that "exhibits the properties of being novel having the value and/or having richness of interpretations” (Rosenman and Gero 1993, 111112). Amongst these different sets of criteria, Sarka and Chakrabati 
conclude that most criteria will list Novelty and Usefulness when defining and measuring creativity. Furthermore, being Surprising is the next most common feature of creative design. For instance, Hayes (1978) and Nguyen and Shanks (2009) argue that creativity should have three main characteristics: Valuable, Novel and Surprising. Maher's (2010) creativity criteria are also Value, Novelty and Unexpectedness. She states that Unexpectedness is one of the creativity feature that based on expectations and depends on both Novelty and Value. From the above review, we can see that three criteria are commonly used for evaluating creativity: Novelty (Innovation), Usefulness (Value), and Surprisingness (Unexpectedness).

The present study evaluates and compares the creativity of outcomes produced from designers working in PDEs and GMEs. Three criteria are adopted for this purpose - Innovation (adapted from novelty), Usefulness and Surprisingness. To this we add an overarching category for the experts to access the outcomes in a more holistic manner - Overall creativity.

\subsection{Creativity in parametric design}

Past research suggests a positive linkage between design creativity 
and parametric design. For example, Hesselgren et al. (2007) argue that parametric design is a new mode of thinking that allows designers to explore the entirety of the design space within the identified constraints. In parametric design, the decomposition of design into rules, and the flexibility of allowing easier changes to the design, is also allegedly beneficial for creative results (Wortmann and Tunçer 2017). Jabi et al. (2017) believe that parametric design might be appropriate for addressing problems with a high level of complexity, thereby encouraging designers to explore a new and unexpected design solution space. This capacity is identified as an important feature in design creativity. As mentioned previously, Iordanova and Tidafi et al. (2009) studied design creativity in parametric design by observing a relatively small group of architectural students using the parametric software GenerativeComponents ${ }^{\mathrm{TM}}$ (GC). They observed the students' design processes from generation of ideas, through to design exploration and evolution of ideas using three design paradigms; direct description, formulation and generation. Based on TTCT, the design evaluation uses criteria including abundance, flexibility, evolution and originality of ideas, as well as efficiency and coherency. Their result shows that abundance is the most positively influenced aspect of creativity in 
PDEs, especially with the generative method because new ideas are emerging simultaneously as variations. This correlates to Abdelsalam’s (2009) claim that “Parametric software enables the designers to direct their creativity to wide range of exploration using 'whatif' scenarios” (Abdelsalam 2009). Furthermore, during the experiment by Iordanova and Tidafi et al., students expressed a positive attitude towards the use of the generative method, highlighting unforeseen results as promoting design creativity.

Using the same methodology, observations of students in a design studio, Schnabel (2007) studies creativity in PDEs. Compared to the previous study, the advantage of Schnabel's study is that there were 30 students involved in the experiment and the studio lasted for a whole semester. Schnabel's study allows for a deeper exploration of design in PDEs through more complex design tasks and longer design duration. It focuses on the parametric dependencies of spatial perception, fabrication and form finding. Although the author believes that parametric design provides opportunities for in-depth comprehension of the design objectives and assists designers in problem solving, he argues that "parametric design opens up a novel set of opportunities” 
(247), although there remains some ambiguity regarding creativity in PDEs.

Another study by Lee et al. (2014) focuses on exploring creativity in parametric design by employing the combined method of protocol analysis and assessment using the Consensual Assessment Technique (CAT) (Amabile 1982). In Lee et al.’s study, three designers’ parametric design process were analysed in detail using protocol analysis and the design results were evaluated by experts. The results of the study suggest that 'analysis' and 'synthesis” are positively related to creativity in PDEs. However, the research did not manage to benchmark the findings (by, for example, comparing to GMEs) and their sample size is limited. Therefore, the results are difficult to be generalized, and they carefully state that the relationship between parametric design and creativity requires further study.

In contrast, other researchers (eg.Salim and Burry 2010) believe that, in some circumstances, parametric design can actually hinder creativity. This is because, with the number of potential parametric variations increasing, flexible changes can be unmanageable. They argue that parametric design tools have certainly assisted creative think- 
ing through rule algorithms. However, it can also decrease design possibilities because of fixed variations. Further, "the more complex the software is, the lower the usability” (Salim and Burry 2010, 490). In order to innovate, parametric designers need to explore their ideas using complementary creative methods (not necessarily parametric).

The present paper extends the past research summarised in this section by providing much-needed empirical evidence with a larger sample size to measure and explore creativity in parametric design. Rather than using observation or interview, we adopt the method of protocol analysis to provide more in-depth analysis of the design process. Protocol analysis is particularly appropriate for developing rich and rigorous data from small sample sizes. Furthermore, by correlating the results of expert evaluation of design outcomes and protocol analysis of design process in two different design environment (undertaken by the same participants), the comparison of the parametric design environment and geometrical modelling environment provides more statistically valid and reliable results. 


\section{Research method}

\subsection{Protocol analysis}

Protocol analysis is a method for turning qualitative verbal and gestural utterances into quantitative data (Ericsson and Simon 1993; Gero and McNeill 1998). According to Akin (1986), a protocol is the record of the behaviour of designers when interacting with various sketches, models, images or notes, or with each other, captured as videos and/or audios. After collecting the protocol data, a coding scheme is developed and applied to categorise the collected data, enabling detailed studies of the design process in the chosen design environment. As Gero and Tang (2001) argue, protocol analysis has become the prevailing experimental technique for exploring the way in which the design process is understood.

Gero’s Function-Behaviour-Structure (FBS) ontology (Gero 1990) provides one of the most common and accepted foundations for coding cognitive design studies in protocol analysis (Gero and Tang 1999; Kan and Gero 2005; Kan and Gero 2009). Researchers argue that it is potentially capable of capturing most of the meaningful cognitive aspects of design with its systematic coding of design processes 
and the transitions in-between. The eight design processes include formulation, analysis, evaluation, synthesis, and reformulation I, II and III (Gero 1990). The FBS ontology, as illustrated in Figure 1 shows the three classes of ontological variables: Function (F), Behaviour (B) and Structure (S). Function (F) represents design intentions or purposes; Behaviour (B) represents the object's derived behaviour (Bs) or expected behaviour from the structure $(B e)$; and Structure $(S)$ represents the components that make up an artefact and their relationships. The FBS ontology, as the basis of a coding scheme, includes two additional design issues that can be expressed. They are Requirements $(R)$ and Descriptions (D). R represents requirements from outside the design itself and D, descriptions, refers to the documentation of the design.

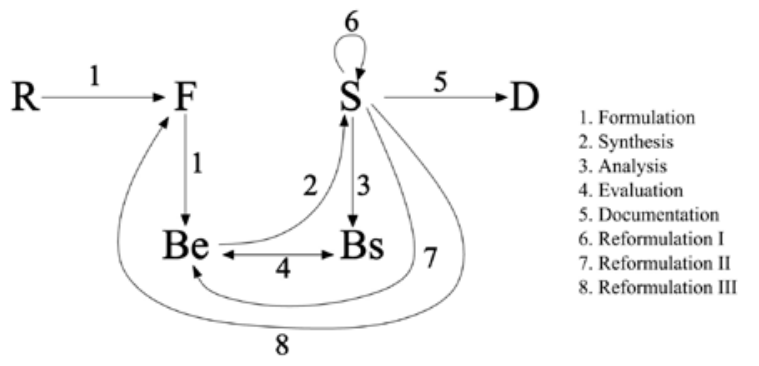

Fig. 1. FBS ontology (Gero 1990) 


\subsection{Jury evaluation}

There is a long history of using jury or expert panel evaluations to assess the creativity of design outcomes. In terms of specific criteria of the evaluation, Sobell and Rothenberg assess sketches by groups of designers using three criteria - “originality," “value” and "overall creative potential” (Sobel and Rothenberg 1980). Getzel and Csikszentmihalyi evaluate drawings of subjects according to "originality," “craftsmanship” and "overall aesthetic value” (Getzels and Csikszentmihalyi 1976). Amabile further develops a formal method of Consensual Assessment Technique (CAT) (Amabile 1982) for evaluating creativity, in which general requirements for both the evaluation process and jury selection are defined. For instance, the products being evaluated should be in random order and the experts should have a certain degree of creative ability themselves, as different views of creativity between experts may have an impact on the final evaluation results. CAT is a relatively mature and accepted assessment method, providing a formal framework for assessing creativity.

The subjective factor in jury evaluation is of course difficult to avoid, as experts tend to assess products according to their own definitions of creativity (Rivlin 1959; Wallen and Stevenson 1960; Eisner 
1965). In order to manage the limitations of subjectivity, it is important to critically define evaluation criteria and provide as much detail as possible. Another important tactic is to select experts with a broad knowledge, who have demonstrated ability in the particular subject area as well as the broad related areas.

\section{Research design}

\subsection{Experiment settings}

Eight designers participated in the experiment, each of whom is a professional architect with an average of eight years of experience in architectural design, and no less than two years' experience using parametric design tools.

The experiment consisted of two design sessions: one session used Rhino as the GME and the other session used Rhino and Grasshopper as the PDE. Each designer was given 40 minutes for an individual design session. Task 1 is a community centre conceptual design and Task 2 is a shopping centre conceptual design, both of similar complexity containing specific functional requirements. A pre-modelled site was provided to each designer prior to their sessions. 
Each experimental session is divided into two parts. In the first part, the participant was required to "think aloud" - to verbalise what s(he) was thinking while designing. A screen capturing programme recorded both their spoken words and actions. If there is insufficient verbal data produced, in the second part of the session a retrospective protocol collection method is used to produce complementary verbal data. That means, after finishing the design task, the recorded videos were played back and each participant was asked to make additional comments about what s(he) was thinking while designing during the session. The data collected, therefore include verbal information about participants' design intentions as well as visual information about their activities.

\subsection{Evaluating creatvity}

The outcomes produced by designers in the experiments were displayed in an online survey and experts were invited to assess the 16 designs according to the given criteria (with scores ranging from 0-7 in each sub-category). Experts were recruited based on the criteria that they must have obtained Architectural degree. Through email invita- 
tion, 19 University postgraduates and staff participated in the evaluating designs from the experiments. The designs were arranged in a random order. Each design was presented as an image and it was accompanied by a paragraph of design description. Figure 2 shows an example web page from the online evaluation, which presents one of the 16 designs to the experts for assessment. There are four main scores to be calculated and integrated, based on Overall creativity, Innovation, Usefulness and Surprisingness. The interpretation of the criteria is described as following:

- Overall creativity: Design represents Innovation, Usefulness and Surprisingness.

- Innovation: Design represents new and original, never known before (Sarkar and Chakrabarti 2011; Sternberg and Lubart 1999)

- Usefulness: A design that is effective at solving a problem

(Nguyen and Shanks 2009), represents ‘appropriateness utility' (Sternberg and Lubart 1999) in terms of 'usefulness, appropriateness and social value’(Nickerson 1994)

- Surprisingness: Design represents unusualness and unexpectedness (Jackson and Messick 1965). 


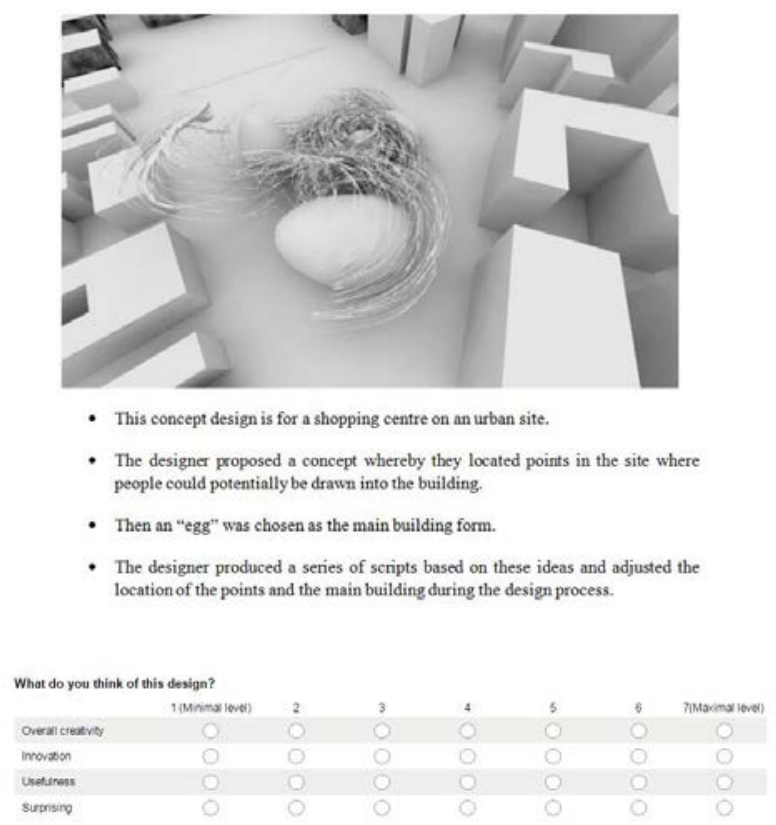

Fig. 2. An example web page of the online evaluation

\section{Results}

\subsection{General observation}

It was apparent in the experiment that the eight designers each showed a clear capacity to understand the design brief and operate the software. While a time limit was used to ensure that the design problems were addressed to a similar level of resolution, some designers stopped at the building massing or façade design stages. However, they all considered site planning as well as the building function in 
some detail, providing a considered response to the conceptual design brief. In both the PDE and the GME, designers started by reading the brief and inspecting the site model provided. During the design process, they also revisited the design brief. The design brief provided some details concerning functional constraints and site conditions. It was up to the designers to decide how many of these conditions to consider in their design.

Through direct observation we found that in the PDE designers tended to build a system of “correct” parametric relationships rather than building a "correct" model. The concept of the whole system seems to be determined at the beginning of the design stage. Designers in the PDE were not completely sure about what would come out after they develop a certain script, and thus there were multiple "Aha” moments, where surprising results emerged. For example, several times designers stated that "this looks good" or "it starts to look interesting," when they referred to the evaluation of their current design situations in the PDE. Designers in the PDE switched between the scripting interface (Grasshopper) and the modelling interface (Rhino) frequently. They often went back to examine the model through the modelling interface after they changed a parameter or parametric relationship, or 
went back to check their previous script definitions using the scripting interface. This inspection of a previous model or script definition can be defined as a kind of perceptual activity, which is connected to the accumulation of generated intentions.

It was also apparent in the experiment that designers exhibit different approaches to parametric design. Some defined and applied rule relationships as the dominant way of exploring and progressing design concepts, others mainly use rules to generate geometries. For example, one designer used the sunlight analysis of the given site model for area planning, which reflects a relatively high-level of conceptual parametric thinking. Another designer generated random points to make a varying façade, which was mainly intended to generate an innovative form. As this study focuses on conceptual design in the parametric environment in general, different approaches to parametric design were observed, which quite closely resemble the actual practices of parametric design.

\subsection{General coding results of design process}

After transcription, two rounds of segmentation (the division of proto- 
cols into individual units based on FBS notions) and coding were conducted. The coding was conducted by one researcher with a time interval of two weeks between the two rounds. Thereafter an arbitration session (to make decisions on any disagreements between the two rounds of coding) was carried out to produce the final protocol, ensuring the precision of the coding. The agreements between the two rounds of coding are $84.77 \%$ (for GME) and 83.48\% (for PDE) respectively. The final arbitrated results were 92.13\% (for GME) and 91.53\% (for PDE). The high level of agreement suggests the reliability of the coding results.

Table 1 shows the normalised design issue distributions in the GME and the PDE. We can see that the two design environments produce similar results in terms of design issue distributions. In particular, some similar trends have been observed, for example, more cognitive effort is expended on the Structure (S) than any others in both environments; this is followed by Behaviour from structure (Bs), Expected behaviour (Be), Function (F), and the least effort is expended on Requirements (R).

Table 1. Normalised design issue distributions in the GME and the PDE (Yu, Ostwald, and Gu 2015) 


\begin{tabular}{c|c|c|c|c|c|c|c|c|c|c}
\hline & \multicolumn{2}{|c|}{ R (\%) } & \multicolumn{2}{c|}{ F (\%) } & \multicolumn{2}{c|}{ Be (\%) } & \multicolumn{2}{c|}{ Bs (\%) } & \multicolumn{2}{c}{ S (\%) } \\
\cline { 2 - 12 } & GME & PDE & GME & PDE & GME & PDE & GME & PDE & GME & PDE \\
\hline Designer 1 & 2.44 & 1.79 & 11.71 & 4.02 & 18.05 & 13.39 & 26.83 & 28.13 & 40.98 & 52.68 \\
\hline Designer 2 & 1.67 & 1.15 & 7.50 & 3.45 & 12.92 & 20.69 & 37.08 & 39.08 & 40.83 & 35.63 \\
\hline Designer 3 & 2.96 & 1.99 & 3.70 & 1.49 & 13.33 & 5.97 & 31.11 & 37.31 & 48.89 & 53.23 \\
\hline Designer 4 & 2.17 & 0.82 & 4.35 & 3.29 & 22.61 & 25.10 & 31.74 & 30.04 & 39.13 & 40.74 \\
\hline Designer 5 & 2.98 & 1.29 & 9.79 & 4.29 & 18.30 & 24.89 & 30.64 & 25.75 & 38.30 & 43.78 \\
\hline Designer 6 & 0.47 & 0.39 & 6.05 & 9.41 & 12.56 & 27.84 & 25.58 & 22.35 & 55.81 & 40.00 \\
\hline Designer 7 & 8.94 & 2.25 & 11.17 & 9.01 & 30.17 & 35.59 & 22.91 & 18.92 & 26.82 & 34.68 \\
\hline Designer 8 & 1.38 & 2.46 & 8.65 & 5.74 & 18.34 & 27.87 & 33.56 & 28.28 & 38.06 & 35.66 \\
\hline Mean & 2.88 & 1.52 & 7.87 & 5.09 & 18.29 & 22.67 & 29.93 & 28.73 & 41.10 & 42.05 \\
\hline SD & 2.59 & 0.72 & 3.00 & 2.81 & 5.93 & 9.26 & 4.59 & 6.86 & 8.47 & 7.40 \\
\hline
\end{tabular}

\subsection{Evaluation results for design outcome}

Table 2 shows selected illustrations of the design outcomes collected from the experiment. Table 3 shows the jury evaluation results of these design outcomes. From the table we can see that the average “Overall creativity” score of designs produced in PDE (4.11) is 4.62\% higher than those produced in GME (3.92). For “innovation,” the average score in PDE (3.84) is 5.47\% higher than its counterpart in GME (3.63), for “surprisingness,” PDE (3.93) is 9.16\% higher than GME (3.57), and finally for "usefulness," interestingly, the average score in GME is $1.27 \%$ higher than the one in PDE. From these results we might infer that PDEs can potentially support creativity in terms of Overall creativity, Innovation and especially Surprisingness. This may 
be due to the powerful form-making capacities in parametric design in comparison with traditional geometric modelling. However, PDEs seem to be less effective in supporting Usefulness than GMEs.

Table 2. Design outcomes collected from the design experiment

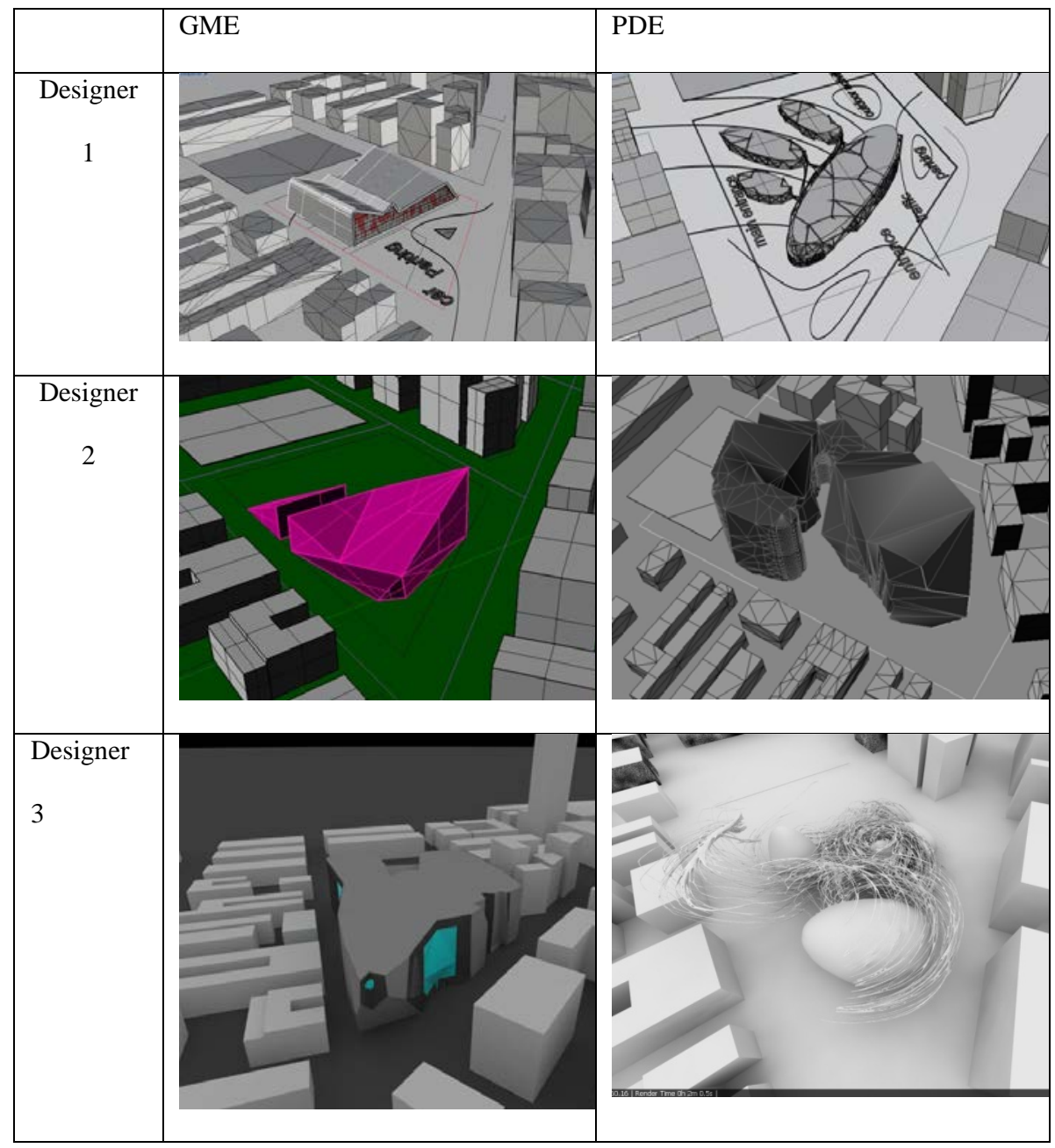




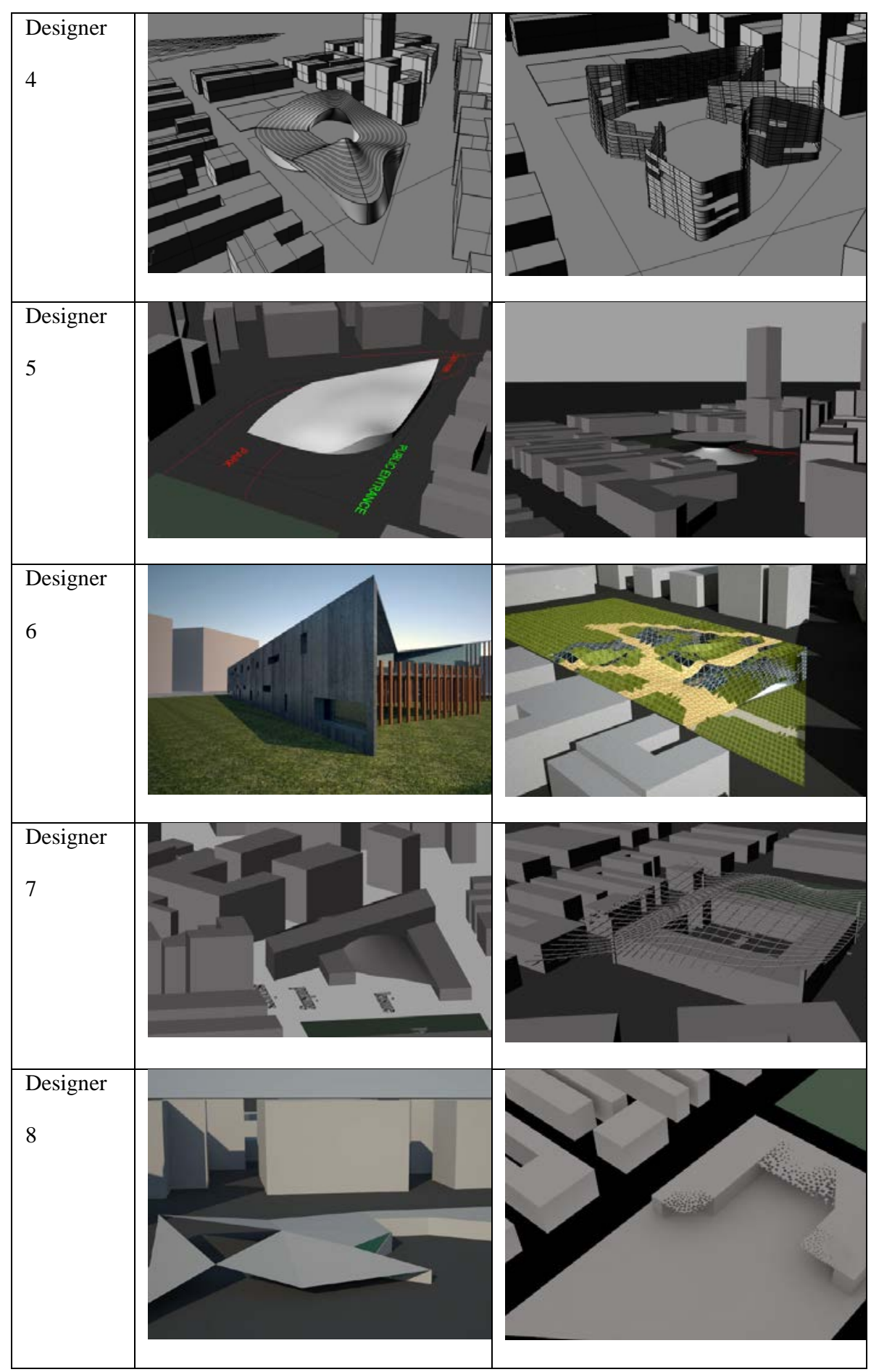


Table 3. Jury evaluation results of the collected design outcomes

\begin{tabular}{|c|c|c|c|c|c|}
\hline \multirow[t]{9}{*}{ GME } & $\begin{array}{l}\text { Average of Jury evalua- } \\
\text { tion results }\end{array}$ & $\begin{array}{l}\text { Overall crea- } \\
\text { tivity }\end{array}$ & $\begin{array}{l}\text { Innova- } \\
\text { tion }\end{array}$ & $\begin{array}{l}\text { Useful- } \\
\text { ness }\end{array}$ & $\begin{array}{l}\text { Surpris- } \\
\text { ingness }\end{array}$ \\
\hline & Designer 1 & 3.58 & 3.16 & 3.53 & 3.11 \\
\hline & Designer 2 & 4.05 & 3.58 & 3.95 & 3.47 \\
\hline & Designer 3 & 3.89 & 3.68 & 3.74 & 3.78 \\
\hline & Designer 4 & 4.32 & 4.21 & 4.22 & 4.11 \\
\hline & Designer 5 & 3.95 & 3.84 & 3.37 & 3.79 \\
\hline & Designer 6 & 3.95 & 3.58 & 4.74 & 3.53 \\
\hline & Designer 7 & 3.05 & 2.95 & 3.53 & 2.79 \\
\hline & Designer 8 & 4.58 & 4.05 & 4.32 & 4.00 \\
\hline \multirow[t]{8}{*}{ PDE } & Designer 1 & 4.58 & 4.11 & 4.26 & 3.68 \\
\hline & Designer 2 & 3.95 & 3.68 & 3.79 & 3.68 \\
\hline & Designer 3 & 4.42 & 4.05 & 3.16 & 4.79 \\
\hline & Designer 4 & 4.05 & 3.74 & 3.11 & 3.79 \\
\hline & Designer 5 & 3.11 & 3.21 & 2.53 & 3.47 \\
\hline & Designer 6 & 4.89 & 4.53 & 5.00 & 4.63 \\
\hline & Designer 7 & 3.89 & 3.79 & 4.84 & 3.63 \\
\hline & Designer 8 & 3.95 & 3.63 & 4.37 & 3.74 \\
\hline \multirow[t]{2}{*}{ GME } & Average & 3.92 & 3.63 & 3.93 & 3.57 \\
\hline & SD & 0.461 & 0.422 & 0.472 & 0.447 \\
\hline \multirow[t]{2}{*}{ PDE } & Average & 4.11 & 3.84 & 3.88 & 3.93 \\
\hline & SD & 0.538 & 0.391 & 0.887 & 0.495 \\
\hline \multicolumn{2}{|r|}{ Differences } & $4.62 \%$ & $5.47 \%$ & $1.27 \%$ & $9.16 \%$ \\
\hline
\end{tabular}




\subsection{Correlation analysis}

To review the results, we firstly grouped the three highest and three lowest evaluation scores in each design environment in terms of Overall creativity, Innovation, Usefulness and Surprisingness (Table 4). For instance, Designers 4, 8 and 2 were grouped into the highest Overall creativity score group in GME while Designers 1, 3 and 7 were in the lowest Overall creativity score group in GME.

Table 4. Grouping of high and low evaluation score groups

\begin{tabular}{l|l|l|l}
\hline & & \multicolumn{1}{|c}{ GME } & PDE \\
\hline Overall creativity & High score group & Designer 4, 8, 2 & Designer 6, 1,3 \\
\cline { 2 - 4 } & Low score group & Designer 7, 1, 3 & Designer 5, 7, 8, 2 \\
\hline Innovation & High score group & Designer 4, 8, 5 & Designer 6, 1, 3 \\
\cline { 2 - 4 } & Low score group & Designer 7, 1, 2, 6 & Designer 5, 2, 8 \\
\hline Usefulness & High score group & Designer 4, 6, 8 & Designer 6, 7, 8 \\
\cline { 2 - 4 } & Low score group & Designer 5, 7, 1 & Designer 5, 4, 2 \\
\hline Surprising & High score group & Designer 4, 8, 5 & Designer 3, 6, 4 \\
\cline { 2 - 4 } & Low score group & Designer 7, 1, 2 & Designer 5, 7, 1, 2 \\
\hline
\end{tabular}

We then examined the corresponding coding results of design process (design issue distribution) for both the high and low evaluation score groups in both design environments. Table 5 shows the 
pairings in term of Overall creativity. Since there are not enough segments in Requirement $(R)$ to produce statistically valid results, only $F$, $B e, B s$ and $S$ are analysed. Differences between the high and low score groups in each design environment are calculated and compared. We set the threshold of $30 \%$ differences as statistically significant. From Table 5 we can see that there is no significant differences in the two groups between the two design environments.

Table 5. Design issue distribution results for both high and low evaluation score groups in terms of Overall creativity

\begin{tabular}{|c|c|c|c|c|c|}
\hline & & F (\%) & Be (\%) & Bs (\%) & S (\%) \\
\hline \multirow[t]{3}{*}{ GME } & $\begin{array}{l}\text { High } \\
\text { Score }\end{array}$ & 6.83 & 17.96 & 34.13 & 39.34 \\
\hline & Low Score & 8.86 & 20.52 & 26.95 & 38.90 \\
\hline & Difference & $\begin{array}{c}- \\
22.87 \%\end{array}$ & $\begin{array}{c}- \\
12.48 \%\end{array}$ & $21.03 \%$ & $1.13 \%$ \\
\hline \multirow[t]{3}{*}{ PDE } & High Score & 4.97 & 15.73 & 29.26 & 48.64 \\
\hline & Low Score & 5.62 & 27.26 & 28.01 & 37.44 \\
\hline & Difference & $\begin{array}{c}- \\
11.55 \%\end{array}$ & $\begin{array}{c}- \\
42.28 \%\end{array}$ & $4.29 \%$ & $23.03 \%$ \\
\hline $\begin{array}{c}\text { PDE } \\
\text { VS. } \\
\text { GME }\end{array}$ & $\begin{array}{l}\text { Difference be- } \\
\text { tween high and } \\
\text { low group }\end{array}$ & $11.32 \%$ & $29.8 \%$ & $16.74 \%$ & $21.9 \%$ \\
\hline
\end{tabular}


In terms of innovation, from Table 6 we can see that the difference of Expected Behaviour (Be) between the PDE (-35.74\%) and the GME (6.71\%) is significant (42.45\%). And in the PDE, Be is higher in low evaluation score group (24.48\%) than high evaluation score group (15.73\%). This suggests that in the PDE designers' activities related to Expected Behaviour (Be) tend to hinder "innovation" while this is not the case in the GME. This may because designers need to allocate extra effort in setting rule algorithm related goals in the PDE, which results in less time and effort in design related goals. This may have caused design outcomes being less “innovative.”

Table 6. Design issue distribution results for both high and low evaluation score groups in terms of Innovation

\begin{tabular}{r|l|r|r|r|r}
\hline \multirow{2}{*}{ GME } & High Score & F (\%) & Be (\%) & Bs (\%) & S (\%) \\
\cline { 2 - 6 } & Low Score & 7.60 & 19.75 & 31.98 & 38.50 \\
\cline { 2 - 6 } & Difference & 9.11 & 18.43 & 28.10 & 41.11 \\
\hline \multirow{2}{*}{ PDE } & High Score & $-16.59 \%$ & $6.71 \%$ & $12.13 \%$ & $-6.36 \%$ \\
\cline { 2 - 6 } & Low Score & 4.97 & 15.73 & 29.26 & 48.64 \\
\cline { 2 - 6 } & Difference & 4.49 & 24.48 & 31.04 & 38.36 \\
& & $9.65 \%$ & $-35.74 \%$ & $-5.71 \%$ & $21.14 \%$ \\
\hline PDE VS. & Difference between high & $26.24 \%$ & $42.45 \%$ & $17.84 \%$ & $27.5 \%$ \\
\cline { 2 - 6 } & and low group & & & & \\
\hline
\end{tabular}


In terms of Usefulness, from Table 7 we can see that the differences of Function (F), Expected Behaviour (Be) and Structure Behaviour (Bs) between the PDE and the GME are significant, which are 96.04\%, 42.14\%, and 38.24\% respectively. This suggests that considering Functions (F), Expected Behaviour (Be) and Structure behaviour (Bs), related issues could have very significant impact on the design outcome in terms of usefulness. Especially Function (F), in the PDE $F$ is significantly higher (54.35\%) in the high evaluation score group (8.05\%) than the low evaluation score group (3.68\%).

Table 7. Design issue distribution results for high and low evaluation score groups in terms of Usefulness

\begin{tabular}{r|l|r|r|r|r}
\hline & & F (\%) & Be (\%) & Bs (\%) & S (\%) \\
\hline GME & High Score & 6.35 & 17.84 & 30.29 & 44.33 \\
\cline { 2 - 6 } & Low Score & 10.89 & 22.17 & 26.79 & 35.37 \\
\cline { 2 - 6 } & Difference & $-41.69 \%$ & $-19.56 \%$ & $11.55 \%$ & $20.23 \%$ \\
\hline PDE & High Score & 8.05 & 30.43 & 23.18 & 36.78 \\
\cline { 2 - 6 } & Low Score & 3.68 & 23.56 & 31.62 & 40.05 \\
\cline { 2 - 6 } & Difference & $54.35 \%$ & $22.58 \%$ & $-26.69 \%$ & $-8.16 \%$ \\
\hline PDE & Difference between & $96.04 \%$ & $42.14 \%$ & $38.24 \%$ & $28.39 \%$ \\
VME & high and low group & & & & \\
\hline
\end{tabular}


In terms of Surprisingness, from Table 8 we can see that the difference of Expected Behaviour (Be) between the PDE (-3.09\%) and the GME (-37.70\%) is significant (34.61\%). This suggests that designers consider Expected Behaviour (Be) differently when designing in the PDE and the GME, which can have an impact on their design outcomes in terms of Surprisingness. In both environments, the low evaluation score group has a higher $B e$, which might mean that this type of activities can potentially hinder the creativity in design outcomes in terms of Surprisingness.

Table 8. Design issue distributions for both high and low evaluation score groups in terms of Surprisingness

\begin{tabular}{r|l|r|r|r|r}
\hline & & F (\%) & Be (\%) & Bs (\%) & S (\%) \\
\hline \multirow{2}{*}{ GME } & High Score & 22.79 & 59.25 & 95.94 & 115.49 \\
\cline { 2 - 6 } & Low Score & 30.38 & 61.14 & 86.82 & 108.63 \\
\cline { 2 - 6 } & Difference & $-24.98 \%$ & $-3.09 \%$ & $9.51 \%$ & $5.94 \%$ \\
\hline \multirow{2}{*}{ PDE } & High Score & 14.19 & 58.91 & 89.70 & 133.97 \\
\cline { 2 - 6 } & Low Score & 20.77 & 94.56 & 111.88 & 166.77 \\
\cline { 2 - 6 } & Difference & $-31.68 \%$ & $-37.70 \%$ & $-19.82 \%$ & $-19.67 \%$ \\
\hline \multirow{2}{*}{ PDE } & Difference between & $6.7 \%$ & $34.61 \%$ & $29.33 \%$ & $25.61 \%$ \\
\hline \multirow{2}{*}{ GME } & high and low group & & & & \\
\hline
\end{tabular}




\section{Conclusion}

This paper presents the results of an experiment comparing design cognition in two environments with assessments of design creativity. By correlating the protocol analysis results for the design process with jury evaluations of design outcomes, we can characterise design in PDEs and in GMEs. The results presented in this research should be understood considering a set of limitations due to various factors. For example, due to the different nature of designing in different software, the same amount of designing activities in PDEs and in GMEs as well as their results and effects could still vary quite significantly. Although our participant selection criteria aim to identify designers who are fluent in both environments, nevertheless in PDEs, experienced parametric designers can take less time in designing by defining and manipulating rule algorithm than direct geometric modelling in GMEs. Thus there can be relative more opportunities for designers to explore alternative strategies and solutions in PDE. Therefore, within the same given time constrain, designers in PDEs may be more likely to produce more creative design outcomes. Considering this, one of our further studies is to refine the experiment design, to compare the 
results in PDEs and GMEs minimising this time difference and limitation. Other limitations of the research include the different design styles of individual designers and the subjective design preference of individual experts, which may have affected the results. However, through the carefully considered selection criteria, this limitation have been minimised and our future extension will include more participants to further improve the statistical validity of the results. While acknowledging these limitations associated with the scope and scale of this study, the main findings of the research are summarised hereafter.

First, parametric design can potentially assist the emergence of design creativity in terms of Overall creativity, Innovation and Surprisingness, and it is especially beneficial for Surprisingness. This finding broadly supports previous research which argues that parametric design can inspire abundance and unforeseen or unpredictable design ideas (Iordanova et al. 2009; Schnabel 2007). However, there are no significant differences observed between the two design environments in terms of Usefulness in creativity evaluation, which means it is less likely to be affected by the design tools used.

Second, there are no significant differences in the distribution of design issues between the high evaluation score group and the 
low evaluation score group in terms of Overall creativity in both design environments. This suggests that design cognition in general does not necessarily affect overall creativity in design.

Third, the distributions of Expected Behaviour (Be) differ significantly between the PDEs and the GMEs, in terms of the Innovation, Usefulness and Surprisingness evaluation. This suggests that this type of activities during parametric design processes is an important influential factor that affects Innovation, Usefulness and Surprisingness in design outcomes. Further, the distributions of Function $(F)$ and Structure Behaviour (Bs) are also significantly different between the PDE and the GME, in terms of Usefulness evaluation, especially for F. This suggests that considering rule-algorithm related functions in the PDE may support design creativity by producing more useful design solutions. For example, the use of building-performance related rulealgorithm settings during parametric design can lead to more useful design solutions.

With the increasing application of parametric design, we can see many new and complex designs emerging, which were not easily achievable before. This research explores creativity in PDEs by comparing to the traditional GMEs, and by correlating the analysis of the 
design process and the expert evaluation of the design outcome. These

findings enhance our understanding of the way in which parametric

design may assist design creativity, and have potential benefits for

both design education and design practice.

\section{References}

Abdelsalam, Mai. 2009. The Use of the Smart Geometry through Various Design Processes: Using the programming platform (parametric features) and generative components. Paper presented at the Proceedings of the Arab Society for Computer Aided Architectural Design (ASCAAD 2009), Manama, Kingdom of Bahrain.

Akin, Omer. 1986. Psychology of Architectural Design Pion, London.

Amabile, T. M. 1982. "Social psychology of creativity: A consensual assessment technique." Journal of Personality and Social Psychology 43:997-1013.

Bilda, Zafer, and Halime Demirkan. 2003. "An insight on designers' sketching activities in traditional versus digital media." Design Studies 24 (1):27-50. doi: 10.1016/s0142694x(02)00032-7.

Bilda, Zafer, John S. Gero, and Terry Purcell. 2006. "To sketch or not to sketch? That is the question." Design Studies 27 (5):587-613. doi: 10.1016/j.destud.2006.02.002.

Black, Alison. 1990. "Visible Planning on Paper and on Screen." Behavior and Info. Technology 9 (4):283-96.

Chien, Sheng-Fen , and Yee-Tai Yeh. 2012. On Creativity and Parametric Design---A preliminary study of designer's behaviour when employing parametricdesign tools. Paper presented at the Proceedings of eCAADe 2012, Czech Republic, 12-14 September 2012.

Csikszentmihalyi, Mihaly. 1988. "Society, culture, and person: a systems view of creativity." In The Nature of creativity : contemporary psychological perspectives, edited by Robert J. Sternberg, 325-39. Cambridge ; New York: Cambridge University Press.

Eisner, E. W. 1965. "A typology of creative behavior in the visual arts." American Educational research journal 2:125-36.

Ericsson, K. Anders, and Herbert A Simon. 1993. Protocol analysis : verbal reports as data. Mass.: MIT Press.

Fallman, Daniel. 2003. Design-oriented human-computer interaction. Paper presented at the Proceedings of the SIGCHI Conference on Human Factors in Computing Systems, Florida, USA.

Gero, J.S. 1990. "Design prototypes: a knowledge representation schema for design." $A I$ Magazine 11 (4):26-36.

Gero, J.S, and T McNeill. 1998. "An approach to the analysis of design protocols." Design Studies 19 (1):21-61. doi: 10.1016/s0142-694x(97)00015-x.

Gero, John, and Hsien-Hui Tang. 1999. Concurrent and Retrospective Protocols and Computer-Aided Architectural Design. Paper presented at the CAADRIA1999, Shanghai.

2001. "The differences between retrospective and concurrent protocols in revealing the process-oriented aspects of the design process." Design Studies 22 (3):283-95. doi: 10.1016/s0142-694x(00)00030-2. 
Getzels, Jacob W, and Mihaly Csikszentmihalyi. 1976. The creative vision : a longitudinal study of problem finding in art. New York: Wiley.

Hayes, J.R. 1978. Cognitive psychology: thinking and creating: Dorsey Press.

Hesselgren, Lars, Renos Charitou, and Stylianos Dritsas. 2007. "The Bishopsgate Tower Case Study." International Journal of Architectural computing 5 (1):61-81. doi: 10.1260/147807707780912912.

Iordanova, Ivanka, Temy Tidafi, Manon Guité, Giovanni De Paoli, and Jacques Lachapelle. 2009. Parametric methods of exploration and creativity during architectural design: A Case study in the design studio. Paper presented at the 13th International Conference on Computer Aided Architectural Design Futures, Montréal.

Jabi, Wassim, Shwe Soe, Peter Theobald, Robert Aish, and Simon Lannon. 2017. "Enhancing parametric design through non-manifold topology." Design Studies 52:96-114. doi: https://doi.org/10.1016/j.destud.2017.04.003.

Jackson, P.W, and S Messick. 1965. "The person, the product and the response: conceptual problems in the assessment of creativity." Journal of personality 33 (3):309-29.

Jordanous, Anna. 2011. Evaluating Evaluation: Assessing Progress in Computational Creativity Research. Paper presented at the Proceedings of the Second International Conference on Computational Creativity (ICCC-11), Mexico City, Mexico

Kan, J. W.T, and J. S Gero. 2009. "Using the FBS ontology to capture semantic design information in design protocol studies." In About: Designing. Analysing Design Meetings, edited by J McDonnell and P Lloyd, 213-29. New York: Taylor \& Francis.

Kan, Jeff W. T, and Jhon S Gero. 2005. Can Entropy Indicate the Richness of Idea Generation in Team Designing? Paper presented at the CAADRIA 2005, New Delhi, India. 2007. Can an objective measurement of design protocols reflect the quality of a design outcome? Paper presented at the Internatinal conference on Engineering design (ICED 2007), Paris, France.

Kaufman, James C, and Robert J Sternberg. 2006. The international handbook of creativity. Cambridge ; New York: Cambridge University Press.

Kim, M.J., and M.L Maher. 2005. "Creative Design and Spatial Cognition in a Tangible User Interface Environment." In Computational and Cognitive Models of Creative Design VI, edited by J Gero and M .L Maher, 233-50. University of Sydney.

Kim, Mi Jeong, and Mary Lou Maher. 2008. "The impact of tangible user interfaces on spatial cognition during collaborative design." Design Studies 29 (3):222-53. doi: 10.1016/j.destud.2007.12.006.

Kolarevic, Branko. 2003. Architecture in the digital age : design and manufacturing. New York, NY: Spon Press.

Lawson, Bryan. 1997. How designers think : the design process demystified. Completely rev. 3rd ed. Oxford ; Boston: Architectural Press.

2002. "CAD and Creativity: Does the Computer Really Help?" Leonardo 35 (3):327-31. doi: 10.1162/002409402760105361.

Lee, Juhyun, Ning Gu, Julie Jupp, and Susan Sherratt. 2012. Towards a formal evaluation of creativity in parametric design process: A pilot study. Paper presented at the Design Research Society conference 2012, Bangkok.

Lee, JuHyun, Ning Gu, and Anthony P. Williams. 2014. "Parametric Design Strategies for the Generation of Creative Designs." International Journal of Architectural computing 12 (3):263-82. doi: 10.1260/1478-0771.12.3.263.

Maher, M .L. 2010. Evaluating Creativity in Humans, Computers, and Collectively Intelligent Systems. Paper presented at the Proceedings of the First DESIRE Network Conference on Creativity and Innovation in Design, Aarhus, Denmark.

Mitchell, W. J. 2003. "Beyond Productivity: Information Technology, Innovation, and Creativity." In National Research Council Washington, D.C. 
Nguyen, Lemai, and Graeme Shanks. 2009. "A framework for understanding creativity in requirements engineering." Information and Software Technology 51 (3):655-62. doi: 10.1016/j.infsof.2008.09.002.

Nickerson, S. R. 1994. "Enhancing creativity." In Handbook of creativity, edited by Robert J. Sternberg. Cambridge: Cambridge University Press.

Oxman, Rivka. 2000. "Design media for the cognitive designer." Automation in Construction 9 (4):337-46. doi: http://dx.doi.org/10.1016/S0926-5805(99)00017-5.

2017. "Thinking difference: Theories and models of parametric design thinking." Design Studies 52:4-39. doi: https://doi.org/10.1016/j.destud.2017.06.001.

Qian, Cheryl Z, Victor Y Chen, and Robert F Woodbury. 2007. Participant Observation Can Discover Design Patterns in Parametric Modeling. Paper presented at the ACADIA2007, Halifax.

Rivlin, L. G. 1959. "Creativity and the self-attitudes and sociability of high school students." Journal of educational psychology 50 (4):147-52. doi: 10.1037/h0045328.

Rosenman, M. A, and J. S Gero. 1993. "Creativity in design using a design prototype approach." In Modeling creativity and knowledge-based creative design, edited by J. S Gero and L. M Maher, 111-38. Hillsdale, New Jersey: Lawrence Erlbaum Associates.

Runco, Mark A, and Steven R Pritzker. 1999. Encyclopedia of creativity. San Diego, Calif.: Academic Press.

Salim, F, and J Burry. 2010. Software Openness: Evaluating Parameters of Parametric Modeling Tools to Support Creativity and Multidisciplinary Design Integration Paper presented at the Proceedings of Computational Science and Its Applications (ICCSA 2010), Berlin and Heidelberg, Germany.

Sarkar, Prabir, and Amaresh Chakrabarti. 2011. "Assessing design creativity." Design Studies 32 (4):348-83. doi: https://doi.org/10.1016/j.destud.2011.01.002.

Schnabel, Marc Aurel. 2007. Parametric Designing in Architecture. Paper presented at the Proceedings of the Twelfth International Conference on Computer Aided Architectural Design Futures, Sydney.

Schön, D.A. 1983. The reflective practitioner: how professionals think in action. New York: Basic Books.

Sobel, R.S, and A Rothenberg. 1980. "Artistic creation as stimulated by superimposed versus separated visual images." Journal of Personality and Social Psychology 39:953-61.

Sternberg, R. J, and T. I Lubart. 1999. "The concept of creativity: prospects and paradigms." In Handbook of creativity edited by Robert J. Sternberg, ix, 490 p. Cambridge, U.K. ; New York: Cambridge University Press.

Tang, H. H, Y. Y Lee, and J. S Gero. 2011. "Comparing collaborative co-located and distributed design processes in digital and traditional sketching environments: A protocol study using the function-behaviour-structure coding scheme." Design Studies 32 (1):1-29. doi: 10.1016/j.destud.2010.06.004.

Torrance, E. 1966. "Torrance Test of creative thinking." In.: Princeton.

Wallen, N. E, and G. M Stevenson. 1960. "Stability and correlates of judged creativity in fifth grade writings." Journal of educational psychology 51:273-6.

Woodbury, R. 2010. Elements of Parametric Design. New York: Routledge.

Wortmann, Thomas, and Bige Tunçer. 2017. "Differentiating parametric design: Digital workflows in contemporary architecture and construction." Design Studies 52:17397. doi: https://doi.org/10.1016/j.destud.2017.05.004.

Yu, Rongrong, Ning Gu, Michael Ostwald, and John Gero. 2015. "Empirical support for problem-solution co-evolution in a parametric design environment." Artificial intelligence for engineering design, analysis, and manufacturing (AIEDAM) 29 (01):33-44. doi: 10.1017/S0890060414000316.

Yu, Rongrong, Michael Ostwald, and Ning Gu. 2015. Empirical evidence of designers' cognitive behavior in a parametric design environment and geometric modeling 
environment. Paper presented at the The International Association of Societies of Design Research Conference (IASDR 2015), Brisbane, Australia. 